\title{
Transcutaneous or Transconjunctival Botulinum Toxin Injection for Thyroid Eye Disease Associated Upper Lid Retraction
}

\section{Sharon She Wan Chow}

Hong Kong University: University of Hong Kong https://orcid.org/0000-0001-8005-1518

Loraine Lok Wan Chow ( $D$ llwchow@hku.hk)

Hong Kong University: University of Hong Kong https://orcid.org/0000-0001-9075-9448 Janice Cheung

C-Mer Dennis Lam \& Partners Eye Centre

Research article

Keywords: Thyroid eye disease, upper lid retraction, botulinum toxin

Posted Date: April 15th, 2021

DOl: https://doi.org/10.21203/rs.3.rs-402516/v1

License: (1) (i) This work is licensed under a Creative Commons Attribution 4.0 International License.

Read Full License 


\section{Abstract}

\section{Background}

Botulinum toxin (BTA) injection has been a standard treatment for thyroid eye disease (TED) related upper lid retraction (ULR), however, currently there is no consensus regarding the method of injection. Both transcutaneous (TC) and transconjunctival (TJ) injection of BTA has been reported to be successful. The aim of this study is to compare the changes between the lid parameters, the rate of complications and the difference in pain scores between the two groups.

Method

A randomized controlled trial was carried out between 09/2017- 07/2019. 22 lids were randomized into either TC and TJ injection. Patients were blinded to receive either 2.5u of TC or TJ BTA injections and were followed up at 1, 3 and 6 months post injection by a masked investigator.

Results

12 and 10 lids were assigned ot the TC and TJ group respectively. At 1 month, the changes of PA were significant in the TJ group $(-3.6 \pm 0.9, p=0.001)$, while the changes of MRD1 were significant in both groups (TC group: $-1.67 \pm 1.74(p=0.02)$, TJ group: $-2.60 \pm 0.89(p=0.003)$ ). At 3 months, the change of MRD2 was only significant in the TJ group $(-1.00 \pm 0.71, p=0.034)$. However, at 6 months, all lid parameter changes from baseline and between 2 groups were statistically insignificant. TC group had a significantly lower $(-1.40 \pm 1.51, p=0.016)$ pain score. There was 1 case of diplopia and 1 case of ptosis in the TC group, while there were 2 cases of ptosis in the TJ group.

Conclusion

At 1 month, the PA in the TJ group and the MRD1 in both groups showed statistically significant changes. At 3 months, the TJ group showed statistically significant changes in MRD2. The pain scores were significantly lower in the TC group. Although at 6 months, there were statistically insignificant changes between the 2 groups, the TJ group showed more promising results in 1 and 3 months, and the TC group has less pain. Hence, we hope to draw a consensus in the treatment of TED associated ULR by recommending the transconjunctival approach for BTA injections, however, we should expect it to be a more painful procedure.

Trial registration

Name of registry: HKU Clinical Trial Registry (HKUCTR)

Trial registration number: HKUCTR-2306

Date of registration: 08 Dec 2017 
URL of trial registry record:

http://www.hkuctr.com/Investigator/Update/876c13c77a1a429e9283423b2d412ee1

\section{Background}

Thyroid eye disease (TED) is an autoimmune systemic disease that affects the thyroid gland as well as orbital soft tissue and extraocular muscles. Ocular manifestations include proptosis, periorbital oedema, chemosis, extraocular movement restrictions as well as upper lid retraction (ULR) ${ }^{1}$. The cause of ULR has been described by various proposed mechanisms. These mechanisms include levator muscle enlargement, contracture or fibrosis, abnormal adhesions between levator and adjacent soft tissue ${ }^{2}$, and increased sympathetic tone of the Muller's muscle ${ }^{3}$. ULR causes functional problems which may lead to dry eye symptoms, exposure keratopathy, corneal ulceration or even visual loss. ULR also leads to aesthetic problems and is often cosmetically unacceptable. Currently, there are many management options for ULR. Surgical option aims at recession of levator muscle, excision of Muller's muscle, introduction of spacers, myotomies and anterior blepharotomies ${ }^{4}$. However, surgical results can be largely unpredictable and may be complicated with over or under-correction and abnormal lid contour, which sometimes leads to multiple surgeries in order to achieve a satisfactory result. Contrastingly, although medical treatment may also cause over or under-correction, the effect is often reversible and it does not affect lid contour. Scott et al first reported the injection of botulinum toxin A (BTA) to the levator muscle as a treatment option for ULR ${ }^{5}$. Since then, many studies have reported the success and effectiveness of injecting BTA for the treatment of ULR, which was able to provide a functionally and cosmetically satisfactory lid position and contour ${ }^{6-9}$. However, to date, there is no consensus regarding the method of injection of BTA for ULR. Both transcutaneous and transconjunctival injection of BTA has been reported to be successful. Shih et $\mathrm{al}^{10}$ and Costa et al ${ }^{11}$ reported a single transcutaneous injection of BTA to be safe and effective and produces adequate lid contour. Nava Castaneda et $\mathrm{al}^{12}$ and Wabbles et al ${ }^{13}$ have also reported transconjunctival injection of BTA to be safe and effective.

The purpose of this study is to prospectively evaluate and compare the effect of transcutaneous and transconjunctival injection of BTA in the treatment TED associated ULR, so to standardize the method of injection, hence maximizing its treatment outcome and minimizing its potential complications. To date, this is the first study evaluating on the method of injection for BTA treatment in TED associated ULR.

\section{Methods}

A randomized controlled trial was carried out from September 2017 - September 2019. The study followed the principles of Declaration of Helsink, approval was obtained from the Institutional Review Board Ethics Committee and all patients gave their informed consent. The study adhered to the CONSORT guidelines.

\section{Patient selection}


The study included a total of 16 patients (26 eyes: 10 bilateral and 6 unilateral). Inclusion criteria include a clinical and laboratory confirmation of the diagnosis of thyroid disease, age older than 18 years old and unilateral or bilateral ULR secondary to TED which has been clinically and hormonally stable for at least 6 months without any ophthalmic signs of active inflammation. Exclusion criteria include a history of orbital decompression due to TED, history of previous orbital or eyelid surgeries, active thyroid status with euthyroidism not achieved at the time of study initiation and not maintained throughout the follow up period, active TED with Mourits' clinical activity score (CAS) $>=4{ }^{14}$, less than 18 years old and presence of sight threatening conditions. All patients underwent a complete ophthalmic examination including slit lamp examination and dilated fundal examination. Baseline parameters including best corrected visual acuity (BCVA), intraocular pressure (IOP), exophthalmometer measurements, tear breakup time (TBUT), Schrimer's test, extraocular movements (EOM), Mourits' CAS were collected. Baseline eyelid parameters were measured in the treatment eye, these include palpebral aperture (PA) which is defined as the widest vertical point between the upper and lower lid margin, upper lid margin reflex distance (MRD1) which is defined as the distance between the upper lid margin and the pupil center, lower lid margin reflex distance (MRD2) which is defined as the distance between the lower lid margin and the pupil center, marginal limbal distance (MLD) which is defined as distance between upper lid margin and sclera-corneal limbus at 12 o'clock at down gaze and levator function (LF) which is defined as the excursion of the upper eyelid from downgaze to upgaze with the frontalis muscle fixed in position by digital pressure. Baseline clinical photo with patient at primary gaze and downgaze positions were taken.

Patients were blinded and assigned to either transcutaneous (TC) injection or transconjunctival (TJ) injection treatment groups according to a randomization allocation sequence. Botulinum toxin A (BTA) (Botox, Allergan Inc., Irvine, CA, USA) injection was administered by one single experience Oculoplastic surgeon in an outpatient basis. Each vial (100U) was diluted in $4 \mathrm{ml}$ of normal saline solution. The concentration obtained was $2.5 \mathrm{U} / 0.1 \mathrm{ml}$. After skin disinfection, a single dose of TBA was injected either transcutaneously (Fig. 1) or transconjunctivally (Fig. 2) by a 30G needle with a tuberculin syringe. For transcutaneous injection, a single transcutaneous injection was administered at the central upper lid area within $3 \mathrm{~mm}$ above the superior tarsal border. For transconjunctival injection, the upper lid was everted and a single transconjunctival injection was administered at the central upper lid area within $3 \mathrm{~mm}$ above the superior tarsal border. A pain score by visual analog scale was obtained from the patient during and $10 \mathrm{~min}$ after injection. Post injection clinical photo was taken at primary gaze and downgaze. All patients were followed up at 2 weeks, 1 month, 3 months and 6 months post injection by a masked investigator who is an Oculoplastic surgeon. At each follow up, parameters including the BCVA, IOP, TBUT, Schirmer's test and EOM were collected. The five eyelid parameters (PA, MRD1, MRD2, MLD, LF) were also obtained. The complication rates including ptosis, diplopia, ecchymosis or ocular discomfort were also recorded at each visit. Post injection clinical photos were also taken at each visit.

\section{Statistical analysis}

All statistical analysis was performed using SPSS (SPSS Inc., Chicago, II.). Continuous variables were expressed as means and standard deviation, data will be compared with student $t$ test. If assumptions of 
normality are not satisfied, the Mann Whitney test will be used instead. Differences were considered statistically significant when $\mathrm{p}$ value $<0.05$.

\section{Results}

\section{Baseline}

26 eyes were divided into 2 groups. There were 15 patients in the TC group and 11 patients in the TJ group. Table 1 shows the baseline demographics between the 2 groups, there were no significant differences between the 2 groups. The mean age of the TC group was $51.0 \pm 10.8$ and the mean age of the TJ group was $58.2 \pm 2.5(p=0.175)$. There were 6 females $(66.7 \%)$ in the TC group and $4(80 \%)$ in the TJ group $(\mathrm{p}=0.597)$.

As for the five eyelid parameters, there were no significant differences between the baseline parameters of the 2 groups (Table 2).

\section{Follow ups}

All patients were followed up at post injection 2 weeks, 1 month, 3 months and 6 months. There were 2 patients in the TJ group who wish to exit the study at post injection 1 month and post injection 3 months respectively. 1 patient opted for definitive surgical correction by anterior blepharotomy, the other patient was not keen for further follow up after injection.

At 2 weeks, both TC and TJ group had improvement in lid parameters but were not significant when compared to their own baseline, and when comparing between TC and TJ groups, the changes from baseline between 2 groups were also clinically insignificant (Table 3 ).

At 1 month, the changes of PA from baseline were significant in both groups (TC: -1.2 (1.5) $p=0.053$, TJ: $-3.6(0.9) p=0.001)$ and when comparing between the 2 groups, the changes were significantly higher in the TJ group ( $p=0.008)$. As for MRD1, the changes from baseline were significant in both groups (TC group: -1.67 (1.73) $p=0.02$, TJ group: $-2.60(0.89), p=0.003)$, however, when comparing between the 2 groups, there was no statistically significant changes (Table 4).

At 3 months, the change of MRD2 from baseline was only significant in the TJ group (-1.00 (0.71), $p=0.034)$. There were no clinically significant changes between the 2 groups (Table 5 ).

At 6 months, all lid parameter changes from baseline and between 2 groups were statistically insignificant (Table 6).

When comparing the TC and TJ group, at 2 weeks, both groups showed improvement but there were no significant differences between the 2 groups. At 1 month, the changes of PA from baseline were significant in both groups and it was significantly higher in the TJ group, while the changes of MRD1 from baseline were significant in both groups but no significance was shown between the 2 groups. At 3 
months, the changes of MRD2 were only significant in the TJ group and there were no statistically significant differences between the 2 groups. However, at 6 months, all lid parameter changes from baseline and between 2 groups were statistically insignificant.

\section{Pain Score}

Regarding pain score, the drop in pain score from during injection to 10 min after injection was significant in the TC group $(-1.40(1.51), p=0.016)$ but there was no statistically significant changes in pain score in the TJ group.

\section{Complications}

Regarding complications (Table 7), in the TC group, at 2 weeks post injection, 1 patient reported diplopia upon upgaze. Upon EOM examination, she was noted to have a mild limitation in elevation in her treated eye, the limitation resolved spontaneously at 1 month post injection (Fig. 5). Also, 1 patient reported ptosis at 2 weeks post injection, which resolved at 1 month post injection (Fig 6). In the TJ group, there were 2 cases of ptosis at post injection 1 week and post injection 2 weeks respectively, which all spontaneously resolved at post injection 1 month. There was no statistically significant difference between the complication rates amongst the 2 groups.

\section{Discussion}

The prevalence of TED in diagnosed cases of Grave's disease is as high as $25-50 \%{ }^{15-17}$. ULR is one of the most common findings in $\mathrm{TED}^{18}$, and it often requires medical attention as it can be both functionally and aesthetically debilitating. Functionally, ULR can lead to ocular exposure, which may cause dry eye problems, ocular irritation, exposure keratopathy, corneal ulcerations, corneal scarring and even vision loss. Aesthetically, it may cause asymmetry in both eyes and may lead to impairment in cosmesis. Several surgical techniques such as levator recession ${ }^{19,20}$, full thickness blepharotomy ${ }^{21}$ and spacers ${ }^{22}$ have been used, although effective results have been shown, these surgical techniques are often unpredictable and can lead to complications such as contour problems, repeated surgeries and wound infection. Moreover, majority of patients prefer less invasive options in the management of ULR. BTA is a neurotoxin that acts on the motor end plates of muscles to temporarily paralyze the muscle by inhibiting the release of local acetylcholine ${ }^{9}$. BTA was first used in the treatment of TED associated ULR in 1984, which achieved a good lid positioning for 30 days $^{5}$. Subsequently, many studies have been carried out on the treatment outcome of BTA treatment for TED associated ULR. A recent literature review on the nonsurgical treatment options for ULR in TED included 10 studies addressing the use of $B T A^{23}$, of which 7 studies $^{7-11,24,25}$ were carried out with transcutaneous BTA injections and 2 studies ${ }^{26,27}$ were carried out with transconjunctival BTA injections and 1 study ${ }^{5}$ did not specify the method of injection. Although BTA has been proven by multiple studies to be an effective treatment option, the method of injection has never been studied. It is necessary to standardize the method of administration of BTA injections in the treatment of TED associated ULR, so to optimize the treatment outcome and to minimize potential 
complications. We believe to be the first to compare and report on the treatment outcomes of the 2 different methods of injection, either transcutaneously or transconjunctivally.

Our study demonstrates improvement in lid parameters in both TC and TJ injections in the early post injection period of 2 weeks but the difference between the 2 groups were not significant. However, at 1 month post injection, the changes of PA from baseline were significant in both TC and TJ groups, and when comparing between the 2 groups, the changes were significantly higher in the TJ group. This is in keeping with the limited studies on transconjunctival BTA injections, where Uddin et al ${ }^{26}$ reported all 11 patients to have some improvement in the amount of lid retraction after transconjunctival BTA injections of which they were only evident from 1 month onwards. At 3 months, the change of MRD2 from baseline was only significant in the TJ group but there were no clinically significant changes between the 2 groups. This may indicate a more long lasting effect in the TJ group than the TC group. At 6 months, BTA effects in both groups have been weaned off and all lid parameter changes from baseline and between 2 groups were statistically insignificant. This is an expected outcome as this correlates with the natural action of the chemodenervation effect of BTA, which is also shown in most studies that BTA effects are temporary

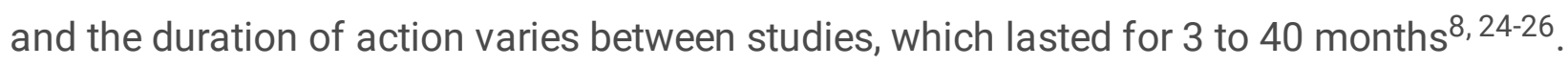

Our study results demonstrate that the TJ group showed more promising results in 1 month post injection, which is usually the most evident phase for BTA effects to take place. TJ group also has a more long lasting effect with an improvement in lid parameters being significant in 3 months post injection. However, the pain score was significantly lower in the TC group.

We propose that transconjunctival injection of BTA may allow for more predictable and long lasting outcome, this is probably due to a more accurate and direct injection of the BTA, as during administration, the upper lid is everted and direct injection of BTA can be administered to the Muller and levator muscles, this method decreases any potential undesirable BTA effects into the orbicularis muscles which is often attained during transcutaneous administration into the levator region as it is a relatively blind procedure.

As for complications, in the TC group, there was 1 case of reported diplopia on upgaze, this was not seen in the TJ group. This can be explained by the lack of superior rectus involvement during transconjunctival administration of BTA, as the injection was placed in the region where the levator aponeurosis inserts onto the upper tarsal plate, but for transcutaneous injection, the relatively blind procedure can allow for injection into the common sheath shared between the levator muscle and the superior rectus, which may cause limitation in upgaze in such cases. Similarly, cases of persistent hypotropias have been reported following BTA injections ${ }^{28}$.

However, our study is limited by the small sample size and short follow up period. Further randomized controlled trials with larger sample sizes and a longer follow up period should be carried out.

\section{Conclusion}


In conclusion, BTA effects in both TC and TJ groups are only significant at 1 month post injection, and TJ group showed a more significant effect. At 3 months, the TJ group continued to show significant effects, which demonstrated a more long lasting outcome. At 6 months, all BTA effects were weaned off in both groups. As for complication rates, there were no statistically significant differences between the 2 groups. As for pain score, the TC group has a significant drop in pain score 10 min after injection. Hence, we hope to draw a consensus in the treatment of TED associated ULR, and we recommend the transconjunctival approach for BTA injections, as it is more effective and more long lasting and does not induce higher complication rates, however, we should expect it to be a more painful procedure.

\section{Abbreviations}

BTA = Botulinum toxin A

$\mathrm{TED}=$ thyroid eye disease

ULR = upper lid retraction

$\mathrm{TC}=$ transcutaneous

$\mathrm{TJ}=$ transconjunctival

CAS = clinical activity score

BCVA = best corrected visual acuity

$\mathrm{IOP}=$ intraocular pressure

TBUT = tear breakup time

EOM = extraocular movements

$\mathrm{PA}=$ palpebral aperture (PA)

$\mathrm{MRD}=$ margin reflex distance

$M L D=$ marginal limbal distance

$L F=$ levator function

\section{Declarations}

\section{Ethics approval}

All procedures performed in studies involving human participants were in accordance with the ethical standards of the institutional and/or national research committee and with the 1964 Helsinki Declaration 
and its later amendments or comparable ethical standards. The study was approved by the Institurional Review Board of the Hospital Authority Hong Kong West Cluster in 10/2017.

\section{Consent for publication}

The participant has consented to the submission of the case report to the journal.

\section{Availability of data and material}

The datasets used and/or analyzed during the current study are available from the corresponding author on reasonable request.

\section{Competing interests}

The authors have no conflicts of interest to declare that are relevant to the content of this article.

\section{Funding}

The authors did not receive support from any organization for the submitted work.

\section{Authors' contribution}

All authors contributed to the study conception and design. Material preparation, data collection and analysis were performed by CSWS and CLWL. The first draft of the manuscript was written by CSWS. All authors read and approved the final manuscript.

\section{Acknowledgements}

Not applicable

\section{Conflict of Interest}

There is no conflict of interest to disclose.

\section{Sources of support}

No sources of support was received for this work.

\section{References}

1. Bahn RS. Graves' ophthalmopathy. N Engl J Med 2010;362:726-38.

2. Small RG. Enlargement of levator palpebrae superioris muscle fibers in Graves' ophthalmopathy. Ophthalmology 1989;96:424-30.

3. Grove AS, Jr. Upper eyelid retraction and Graves' disease. Ophthalmology 1981;88:499-506. 
4. Kazim M, Gold KG. A review of surgical techniques to correct upper eyelid retraction associated with thyroid eye disease. Curr Opin Ophthalmol 2011;22:391-3.

5. Scott AB. Injection treatment of endocrine orbital myopathy. Doc Ophthalmol 1984;58:141-5.

6. Dintelmann T, Sold J, Grehn F. [Botulinum toxin injection-treatment of upper lid retraction in thyroid eye disease]. Ophthalmologe 2005;102:247-50.

7. Ebner R. Botulinum toxin type A in upper lid retraction of Graves' ophthalmopathy. J Clin Neuroophthalmol 1993;13:258-61.

8. Ozkan SB, Can D, Soylev MF, Arsan AK, Duman S. Chemodenervation in treatment of upper eyelid retraction. Ophthalmologica 1997;211:387-90.

9. Traisk F, Tallstedt L. Thyroid associated ophthalmopathy: botulinum toxin A in the treatment of upper eyelid retraction-a pilot study. Acta Ophthalmol Scand 2001;79:585-8.

10. Shih MJ, Liao SL, Lu HY. A single transcutaneous injection with Botox for dysthyroid lid retraction. Eye (Lond) 2004;18:466-9.

11. Costa PG, Saraiva FP, Pereira IC, Monteiro ML, Matayoshi S. Comparative study of Botox injection treatment for upper eyelid retraction with 6-month follow-up in patients with thyroid eye disease in the congestive or fibrotic stage. Eye (Lond) 2009;23:767-73.

12. Nava Castaneda A, Tovilla Canales JL, Garnica Hayashi L, Velasco YLA. [Management of upper eyelid retraction associated with dysthyroid orbitopathy during the acute inflammatory phase with botulinum toxin type A]. J Fr Ophtalmol 2017;40:279-284.

13. Wabbels B, Forl M. [Botulinum toxin treatment for crocodile tears, spastic entropion and for dysthyroid upper eyelid retraction]. Ophthalmologe 2007;104:771-6.

14. Mourits MP, Prummel MF, Wiersinga WM, Koornneef L. Clinical activity score as a guide in the management of patients with Graves' ophthalmopathy. Clin Endocrinol (Oxf) 1997;47:9-14.

15. Piantanida E, Tanda ML, Lai A, Sassi L, Bartalena L. Prevalence and natural history of Graves' orbitopathy in the XXI century. J Endocrinol Invest 2013;36:444-9.

16. Bartalena L, Fatourechi V. Extrathyroidal manifestations of Graves' disease: a 2014 update. J Endocrinol Invest 2014;37:691-700.

17. Hiromatsu Y, Eguchi H, Tani J, Kasaoka M, Teshima Y. Graves' ophthalmopathy: epidemiology and natural history. Intern Med 2014;53:353-60.

18. Frueh BR, Musch DC, Garber FW. Lid retraction and levator aponeurosis defects in Graves' eye disease. Ophthalmic Surg 1986;17:216-20.

19. Moran RE. The correction of exophthalmos and levator spasm. Plast Reconstr Surg (1946) 1956;18:411-26.

20. Harvey JT, Anderson RL. The aponeurotic approach to eyelid retraction. Ophthalmology 1981;88:51324.

21. Elner VM, Hassan AS, Frueh BR. Graded full-thickness anterior blepharotomy for upper eyelid retraction. Trans Am Ophthalmol Soc 2003;101:67-73; discussion 73-5. 
22. Callahan A. Levator Recession with Reattachment to the Tarsus with Collagen Film. Arch Ophthalmol 1965;73:800-2.

23. Grisolia ABD, Couso RC, Matayoshi S, Douglas RS, Briceno CA. Non-surgical treatment for eyelid retraction in thyroid eye disease (TED). Br J Ophthalmol 2017.

24. Salour H, Bagheri B, Aletaha M, et al. Transcutaneous dysport injection for treatment of upper eyelid retraction associated with thyroid eye disease. Orbit 2010;29:114-8.

25. Biglan AW. Control of eyelid retraction associated with Graves' disease with botulinum A toxin. Ophthalmic Surg 1994;25:186-8.

26. Uddin JM, Davies PD. Treatment of upper eyelid retraction associated with thyroid eye disease with subconjunctival botulinum toxin injection. Ophthalmology 2002;109:1183-7.

27. Morgenstern KE, Evanchan J, Foster JA, et al. Botulinum toxin type a for dysthyroid upper eyelid retraction. Ophthal Plast Reconstr Surg 2004;20:181-5.

28. Heyworth PL, Lee JP. Persisting hypotropias following protective ptosis induced by botulinum neurotoxin. Eye (Lond) 1994;8 ( Pt 5):511-5.

\section{Tables}

Table 1: Baseline demographics between TC and TJ groups

\begin{tabular}{|llll|}
\hline & TC group & TJ group & $p$-value \\
\hline Bilateral ULR, $\mathbf{n}(\%)$ & $6(66.7 \%)$ & $2(40.0 \%)$ & 0.334 \\
\hline Age, mean $\pm S D$ & $51.0 \pm 10.8$ & $58.2 \pm 2.5$ & 0.175 \\
\hline Gender, female, $\mathbf{n}(\%)$ & $6(66.7 \%)$ & $4(80.0 \%)$ & 0.597 \\
\hline BCVA, mean $\pm S D$ & $0.88 \pm 0.19$ & $1.00 \pm 0.25$ & 0.232 \\
\hline IOP, mean $\pm S D$ & $18.3 \pm 2.7$ & $17.3 \pm 2.6$ & 0.434 \\
\hline TBUT, mean $\pm S D$ & $4.56 \pm 4.00$ & $3.43 \pm 1.13$ & 0.485 \\
\hline Schirmer's test, mean $\pm S D$ & $10.5 \pm 4.8$ & $6.7 \pm 2.7$ & 0.086 \\
\hline CAS, mean $\pm S D$ & $0.00 \pm 0.00$ & $0.00 \pm 0.00$ & N/A \\
\hline
\end{tabular}

Table 2: Baseline 5 eyelid parameters between TC group and TJ group 


\begin{tabular}{|llll|}
\hline & TC group (mean $\pm S D)$ & TJ group (mean $\pm S D)$ & $p$-value \\
\hline PA & $12.1 \pm 2.4$ & $12.3 \pm 1.1$ & 0.847 \\
\hline MRD1 & $5.93 \pm 2.22$ & $6 . .14 \pm 1.22$ & 0.819 \\
\hline MRD2 & $5.87 \pm 0.99$ & $6.00 \pm 0.82$ & 0.760 \\
\hline MLD & $-1.10 \pm 1.61$ & $-0.29 \pm 1.80$ & 0.298 \\
\hline LF & $12.8 \pm 1.7$ & $12.3 \pm 1.3$ & 0.476 \\
\hline
\end{tabular}

Table 3: Changes from baseline between TC and TJ group at 2 weeks

\begin{tabular}{|c|c|c|c|}
\hline $\begin{array}{l}2 \\
\text { weeks }\end{array}$ & $\begin{array}{l}\text { TC group changes from baseline (mean } \\
\text { (SD)) }\end{array}$ & $\begin{array}{l}\text { TJ group changes from baseline (mean } \\
\text { (SD)) }\end{array}$ & $\begin{array}{l}p- \\
\text { value }\end{array}$ \\
\hline PA & $-1.1(1.8)$ & $-1.0(2.2)$ & 0.920 \\
\hline MRD1 & $-1.44(1.67)$ & $-0.60(1.34)$ & 0.353 \\
\hline MRD2 & $0.00(1.32)$ & $-0.20(0.45)$ & 0.752 \\
\hline MLD & $-0.44(0.73)$ & $0.00(0.00)$ & 0.104 \\
\hline LF & $-0.6(1.7)$ & $0.00(0.00)$ & 0.478 \\
\hline
\end{tabular}

Table 4: Changes from baseline between TC and TJ group at 1 month

\begin{tabular}{|llll|}
\hline $\begin{array}{l}\text { month } \\
\text { mon }\end{array}$ & $\begin{array}{l}\text { TC group changes from baseline } \\
\text { (mean (SD)) }\end{array}$ & $\begin{array}{l}\text { TJ group changes from baseline (mean } \\
(\mathrm{SD}))\end{array}$ & $\begin{array}{l}\boldsymbol{p} \text { - } \\
\text { value }\end{array}$ \\
\hline PA & $-1.2(1.5)$ & $-3.6(0.9)$ & 0.008 \\
\hline MRD1 & $-1.67(1.73)$ & $-2.60(0.89)$ & 0.288 \\
\hline MRD2 & $-0.22(1.20)$ & $-1.00(1.00)$ & 0.778 \\
\hline MLD & $-0.67(0.71)$ & $-0.40(1.14)$ & 0.595 \\
\hline LF & $0.00(2.3)$ & $-0.4(1.1)$ & 0.724 \\
\hline
\end{tabular}

Table 5: Changes from baseline between TC and TJ group at 3 months 


\begin{tabular}{|llll|}
\hline $\begin{array}{l}3 \\
\text { months }\end{array}$ & $\begin{array}{l}\text { TC group changes from baseline } \\
\text { (mean (SD)) }\end{array}$ & $\begin{array}{l}\text { TJ group changes from baseline } \\
\text { (mean (SD)) }\end{array}$ & $\begin{array}{l}\boldsymbol{p} \text { - } \\
\text { value }\end{array}$ \\
\hline PA & $-0.6(1.1)$ & $-1.4(1.3)$ & 0.259 \\
\hline MRD1 & $-1.00(2.18)$ & $-0.40(1.82)$ & 0.612 \\
\hline MRD2 & $-0.22(1.39)$ & $-1.00(0.71)$ & 0.271 \\
\hline MLD & $-0.50(1.17)$ & $0.60(2.41)$ & 0.380 \\
\hline LF & $0.70(2.70)$ & $0.40(0.90)$ & 0.836 \\
\hline
\end{tabular}

Table 6: Changes from baseline between TC and TJ group at 6 months

\begin{tabular}{|llll|}
\hline $\begin{array}{l}\text { months } \\
\text { mo }\end{array}$ & $\begin{array}{l}\text { TC group changes from baseline } \\
\text { (mean (SD)) }\end{array}$ & $\begin{array}{l}\text { TJ group changes from baseline } \\
\text { (mean (SD)) }\end{array}$ & $\begin{array}{l}\boldsymbol{p} \text { - } \\
\text { value }\end{array}$ \\
\hline PA & $0.40(1.00)$ & $-0.80(1.30)$ & 0.078 \\
\hline MRD1 & $0.22(1.30)$ & $-0.20(0.84)$ & 0.529 \\
\hline MRD2 & $-0.67(2.78)$ & $-0.80(1.10)$ & 0.921 \\
\hline MLD & $0.28(3.03)$ & $-0.20(1.64)$ & 0.752 \\
\hline LF & $0.90(1.60)$ & $0.20(0.40)$ & 0.376 \\
\hline
\end{tabular}

Table 7: Complication rates in TC and $\mathrm{TJ}$ groups

\begin{tabular}{|lll|}
\hline & TC group & TJ group \\
\hline Diplopia & $1(11.1 \%)$ & 0 \\
\hline Ptosis & $1(11.1 \%)$ & $2(40.0 \%)$ \\
\hline Ecchymosis & 0 & 0 \\
\hline Occular discomfort & 0 & 0 \\
\hline
\end{tabular}

\section{Figures}




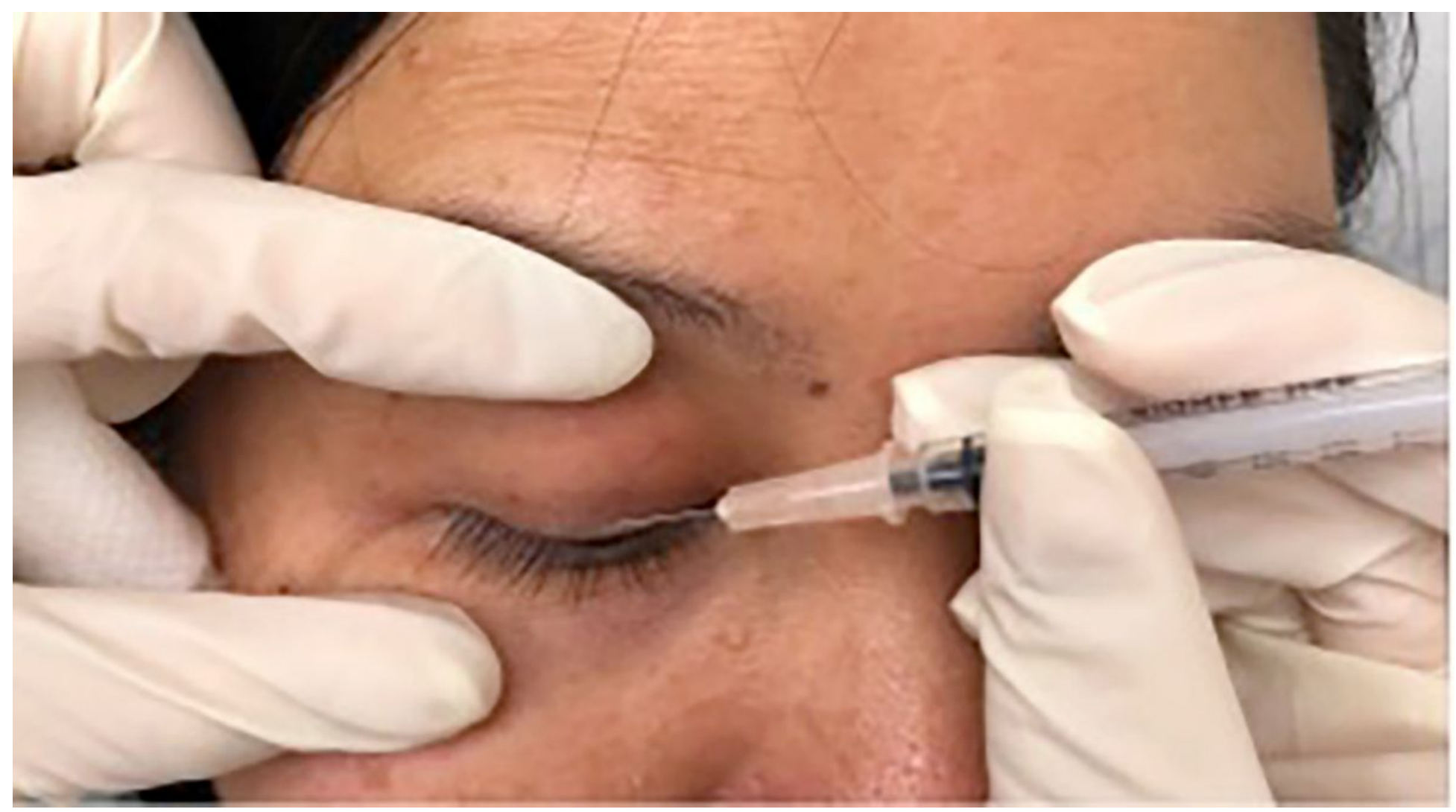

\section{Figure 1}

Transcutaneous injection of BTA

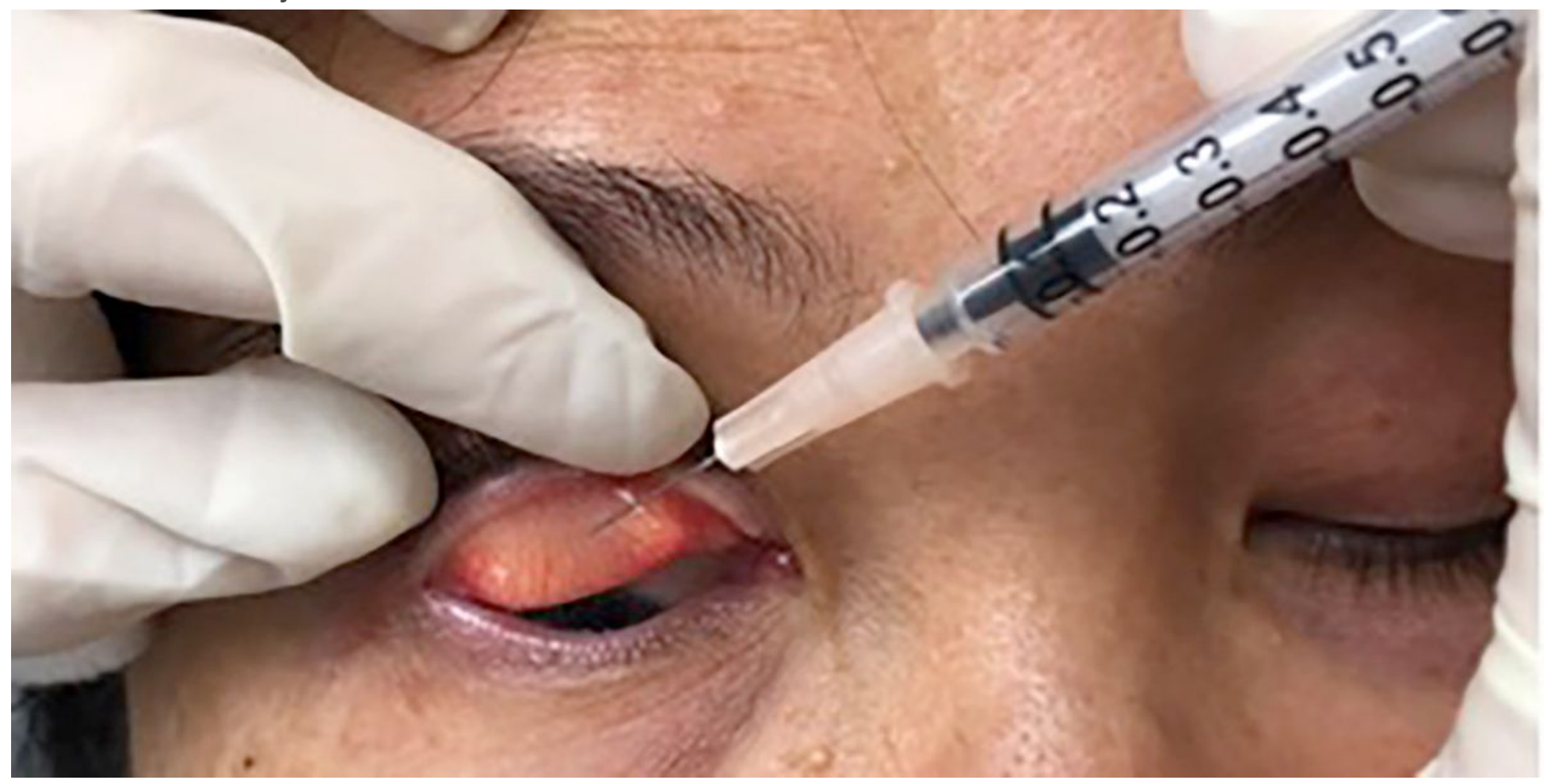

Figure 2

Transconjunctival injection of BTA 

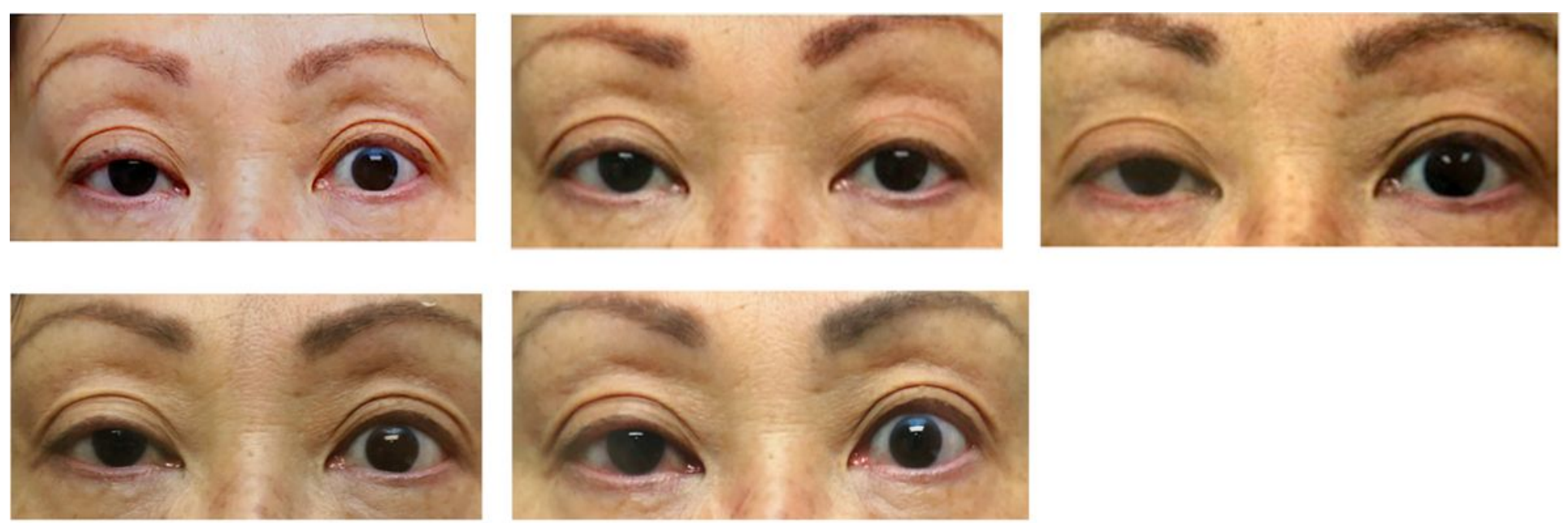

\section{Figure 3}

a-e shows the series of clinical photos of patient A in the TC group, the treatment eye is the left eye: (a) baseline (b) 2 weeks post injection (c) 1 month post injection (d) 3 months post injection (e) 6 months post injection
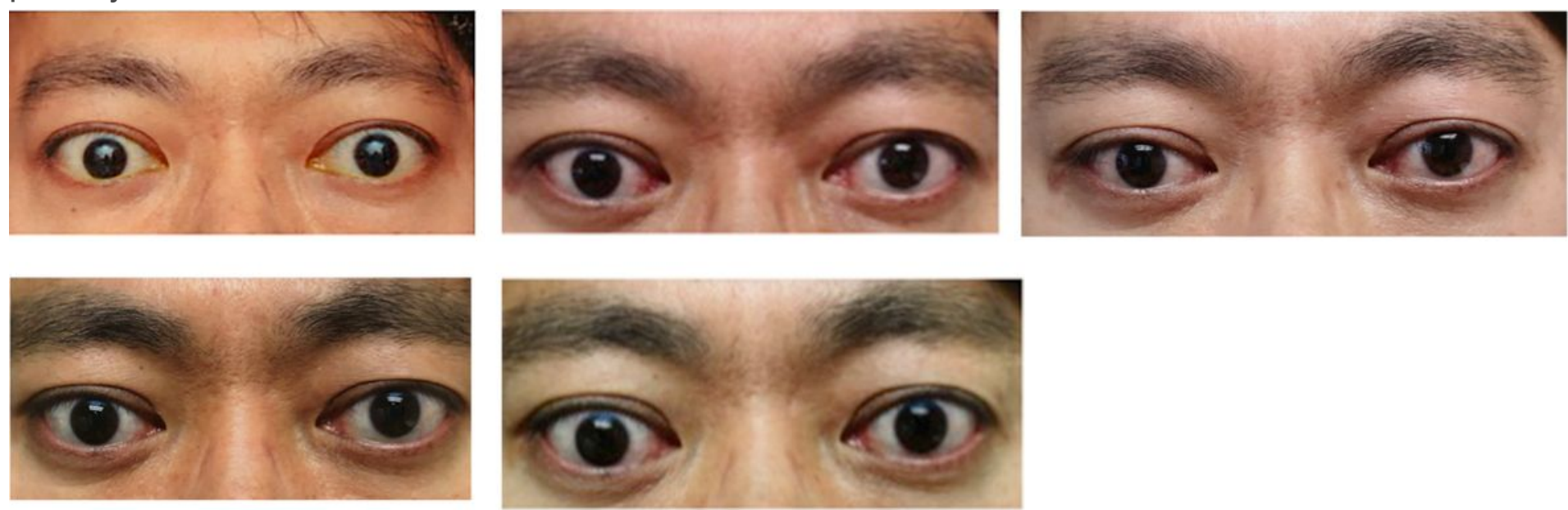

\section{Figure 4}

a-e shows the series of clinical photos of patient B in the TJ group, the treatment eyes are both eyes: (a) baseline (b) 2 weeks post injection (c) 1 month post injection (d) 3 months post injection (e) 6 months post injection 


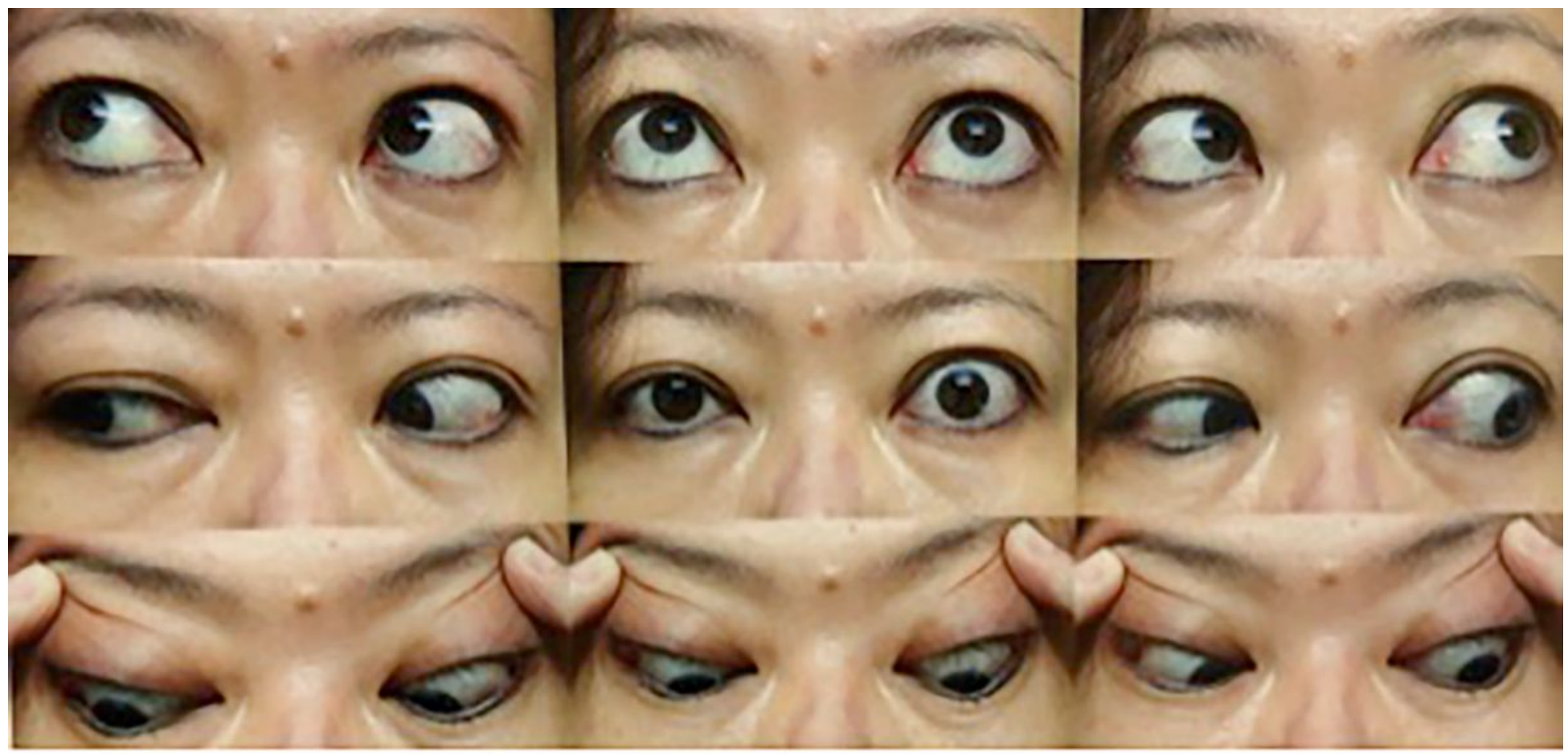

\section{Figure 5}

Limitation in elevation in a patient in the TC group (treated eye: left eye)

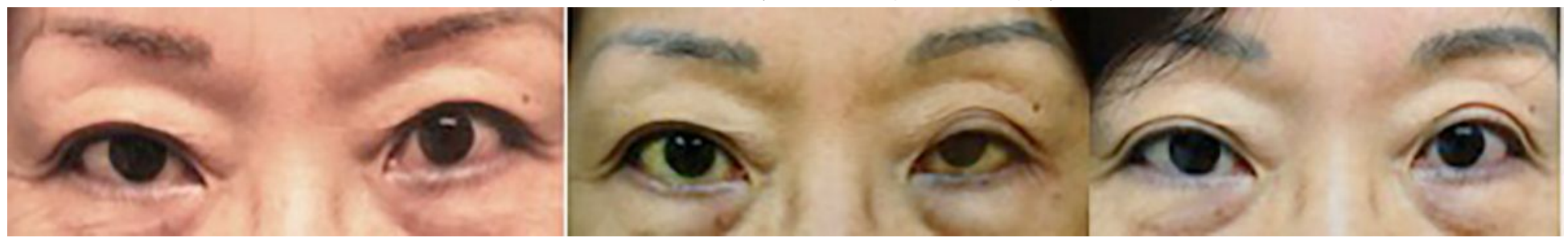

\section{Figure 6}

Clinical photo at baseline, 2 weeks post injection with ptosis, 1 month post injection where ptosis has spontaneously resolved (treated eye: left eye)

\section{Supplementary Files}

This is a list of supplementary files associated with this preprint. Click to download.

- CONSORT2010Checklist.doc 